\title{
Important pharmacogenomic aspects in the management of HIV/AIDS
}

\author{
A Marais, ${ }^{1,2}$ E Osuch, ${ }^{1}$ V Steenkamp, ${ }^{2}$ L Ledwaba ${ }^{1,2}$ \\ 'Department of Pharmacology \& Therapeutics' School of Medicine, Sefako Makghato Health Sciences University' South Africa \\ 2 Department of Pharmacology, School of Medicine, Faculty of Health Sciences, University of Pretoria' South Africa \\ *Corresponding author, email:dramarais@gmail.com / andre.marais@up.ac.za
}

In managing HIV/AIDS with highly active antiretroviral agents, the historical therapeutic aim remains to maintain the plasma concentrations at a level above the half maximal inhibitory concentration (IC50) required for $50 \%$ inhibition in viral replication. Concentration dependent toxicity is often observed in patients with elevated drug exposure and high peak plasma levels in lieu of accurately calculated drug dosages. Similarly low plasma concentrations are frequently witnessed in individuals receiving adequate dosage regimens. Pharmacogenetic variations in drug metabolizing enzymes may contribute to this phenomenon. Over the last decade, knowledge about the role of pharmacogenetics in the treatment and prediction of ARV plasma levels have increased significantly. However, the extent of these genetic variations remain largely unknown in the South African population, which has sparked a renewed enthusiasm for local pharmacogenetic studies.

Keywords: CYP450, genetic polymorphisms, nucleoside reverse transcriptase inhibitors, non-nucleoside reverse transcriptase inhibitors, protease inhibitors

\section{Introduction}

Charles DeLisi and Robert Sinsheimer from the Washington University in St Louis, USA were the first researchers to propose that the human genome was the key in placing the only defensible constraint on biological complexity. They were instrumental in the establishment of the US National Research Council (NRC) committee on mapping and sequencing of the human genome, which commenced in 1988. ' Upon official completion of the Human Genome Project in April 2003, the precise specification, chromosomal locations and molecular composition of all the genes and regulatory elements comprising the human genome had been identified. It continues to serve as an ongoing stimulus in distinct fields of research; ranging from theoretical chemistry to computer technology. Knowledge regarding the human genome has proven beneficial in medical advancement and increased economic competitiveness, including the exponential pace at which personalised or precision medicine is evolving. ${ }^{2,3}$ The importance of genomic research is evident in the Precision Medicine Initiative created in 2015 by former US president Barack Obama, where $\$ 1.45$ billion was allocated to the National Institutes of Health $(\mathrm{NIH})$ and other research centres tasked to improve medical care by individualizing treatment based on an individual's genetic make-up. ${ }^{4}$ However, personalized medicine (at this stage) does not entail the creation of drugs that are unique to a particular patient, but rather compliment the ability to classify an individual into a sub-population that differs in the response to specific treatment or susceptibility to a certain disease. By identifying and classifying patients according to their genomic differences, pharmacological treatment may be directed (and personalized) towards those who will experience the maximum intended benefit, while limiting the expense and side effects for those who will not. ${ }^{5}$

\section{Genetic polymorphisms and drug response}

Approximately $80 \%$ of all drugs are metabolized by the hepatic cytochrome P450 enzyme system, of which the phase 1 metabolism iso-forms CYP1A2 (8.9\%), CYP2C9 (12.8\%), CYP2C19 (6.8\%), CYP2D6 (20\%), and CYP3A4/CYP3A5 (30.2\%) are the most important. ${ }^{6}$ Each CYP450 enzyme is encoded for by a specific gene, which in turn is determined by inherited alleles - one from each parent. These alleles contribute to the phenotype (observable characteristics) of the individual, and may either be dominant or recessive. ${ }^{7}$ When heterozygous alleles are present, the dominant allele will determine the phenotype. Alleles occurring most commonly in the general population are known as "wild type" (or normal), whereas "variant" (or mutation) recessive alleles will only determine the phenotype if a homozygous combination is present. Sequence variations (or Single Nucleotide Polymorphisms/variations SNPs or SNVs) may occur when a variant allele replaces one or both wild-type alleles. Every individual SNV is allocated a unique reference SNP ID number (rs\#) by the HUGO Gene Nomenclature Committee (HGNC) - established by the US National Human Genome Research institute and the Wellcome Trust, to ensure unambiguous reference to genes in scientific communications. ${ }^{8}$ Variant alleles usually encode an enzyme or protein that has reduced (or no) activity, resulting in phenotypical changes which may have an altered effect on drug metabolism or response. ${ }^{9}$

An "extensive metabolizer" phenotype is considered to have normal metabolic activity, where such an individual harbours two copies of the wild-type alleles. In contrast, people with two 
copies of the variant alleles have very little functional metabolic activity, and are classified as "poor metabolizers". Individuals with one wild-type and one variant-type allele have variable or reduced metabolic activity and are referred to as "intermediate metabolizers". Poor and intermediate metabolizers therefore have a much greater risk of drug toxicity since metabolism is impaired on a genetic basis, even if recommended dosage protocols are adhered to. In addition, individuals may inherit multiple copies of wild-type alleles, which result in excess metabolic activity. This phenotype is referred to as "ultra-rapid metabolizers" in which normal therapeutic dosages will not emanate in any (or very little) clinical effect due to the drug being metabolized before adequate plasma concentrations could be reached. ${ }^{10}$

\section{The role of pharmacogenetics in anti-retroviral (ARV) treatment failure or adverse effects}

As is true for most pharmacological entities, the majority of ARVs are metabolized by different cytochrome P450 iso-enzymes. To date, nearly all pharmacogenetic studies have focussed on plasma concentrations of antiretroviral agents by assessing variations in drug metabolizing enzymes and drug transporters. ${ }^{11-15}$ The principle commonality of these studies is based on evaluating genetic polymorphisms which may be responsible for the interindividual pharmacokinetic discrepancies observed in drug exposure and response, and are useful in predicting toxicity and individualized dosing requirements. ${ }^{16}$ These pharmacogenetic studies have largely been performed in Caucasian and Asian populations, and are less inclusive of the African populations. ${ }^{17}$ The literature remains scanty in reporting the effects of these identified polymorphisms which may impact the efficacy and clinical outcomes, such as virological or immunological failure (or success), in patients receiving antiretroviral therapy. ${ }^{18}$ Complex diseases, such as HIV, rely on a combination of diverse gene variants for ARV metabolism (and possibly efficacy) by the CYP450 enzyme system. These genetic variations differ between individuals within a population on both an interindividual and interethnic level, thus providing a possible explanation for the difference in clinical response. ${ }^{19}$ While many international guidelines regarding the initiation and treatment of HIV are available, the South African first line regimens for treatment of naive patients include administration of two nucleoside reverse transcriptase inhibitors (NRTI) (tenofovir, emtricitabine, or lamivudine) in combination with one non-nucleoside reverse transcriptase inhibitor (NNRTI) (efavirenz, nevirapine, dolutegravir or rilpivirine). Second line regimens consist of two NRTI's (including zidovudine) in combination with a protease inhibitor (PI) (ritonavir, lopinavir or atazanavir). ${ }^{20,21}$ Although first line therapy is effective for the majority of South African patients, it is estimated that immunological or virological treatment failure still occurs in approximately $14 \%$ within five years of commencing treatment, resulting in these patients being veered to second line options. ${ }^{22}$

\section{Nucleoside reverse transcriptase inhibitors}

The intracellular pharmacology of the NRTIs is a major determinant of their activity and toxicity, as these drugs are not metabolised by the hepatic CYP450 enzyme system. Tenofovir acts as an adenosine monophosphate nucleoside analogue resulting in the inhibition of HIV replication by causing DNA chain termination. It is metabolised intracellularly through phosphorylation by cellular adenylate kinase and excreted by the kidneys through glomerular filtration and active tubular secretion. ${ }^{23}$ Several pharmacogenetic studies assessing the variants in the $A B C C 4$ gene, commonly implicated in multidrug resistance, were unable to show any association between tenofovir resistance and protein expression in Caucasian populations. ${ }^{24}$ Emtricitabine similarly results in DNA chain termination and thus inhibition of HIV replication. It acts as nucleoside analogue of cytosine and likewise phosphorylated by intracellular enzymes independent of the hepatic CYP450 enzyme system. ${ }^{25}$ Applicable pharmacogenetic evidence is absent in studies concerning emtricitabine.

Lamivudine is phosphorylated by three different enzyme kinases, of which deoxycytidine kinase (dCK) is considered the most important as it is responsible for initiating the activation process to the active form. ${ }^{26}$ Pharmacogenetic studies on lamivudine are largely restricted to polymorphisms of the dCK enzyme in HIV positive Caucasians and different Asian populations in combination with chronic hepatitis B infection. ${ }^{27}$ These studies fail to show a relationship between dCK polymorphisms and treatment success in relation to viral load, CD4+ count or HIV staging.

Zidovudine is structurally related to the thymidine nucleoside and its mechanism of action relates to other NRTIs whereby it prevents incorporation of thymidine into the viral DNA by reverse transcriptase followed by chain termination. Like lamivudine, zidovudine has three important metabolic pathways. Whereas intracellular lymphocytic phosphorylation is responsible for the antiviral action of all NRTIs, the predominant metabolic pathway for zidovudine metabolism is glucuronidation catalysed by the enzyme UDP-glucoronyl transferase. ${ }^{28}$ Pharmacogenetic studies evaluating the UGT2B7 gene partly responsible for zidovudine metabolism, found that African individuals carrying a polymorphism (rs12233719) of this gene, had an almost $200 \%$ increase in the clearance compared to non-carriers. This may explain the high interindividual variability in zidovudine clearance and plasma concentrations..$^{29}$

\section{Non-nucleoside reverse transcriptase inhibitors}

The first generation NNRTIs (efavirenz and nevirapine) form part of the cornerstone in managing HIV-1 infection. The discovery of second generation agents (dolutegravir and rilpivirine) has enhanced the genetic barrier to developing drug resistance and treatment success in combination with other antiretroviral drugs. $^{30}$ Efavirenz is one of the most frequently studied antiretroviral agents concerning genetic polymorphisms relating to its complicated metabolism. The principal catalysts in efavirenz metabolism occur through CYP2A6 and CYP2B6. ${ }^{31}$ The presence of SNP rs28399454, rs28399433 and rs3745274 variant alleles in HIV positive African patients has shown almost a doubling in the plasma concentration of efavirenz when present. Harbouring the SNP rs28399499 was however associated with lower plasma 
concentrations. ${ }^{11}$ Similar results were noticed in a South African study in which the presence of another SNP (rs4803419) was identified as being an independent predictor of efavirenz trough concentrations. $^{32}$

Unlike efavirenz, nevirapine is non-teratogenic and used for the prevention of mother to child transmission. It is primarily metabolized by CYP2B6 and CYP2C19. Several polymorphisms influencing the metabolism by these cytochrome enzymes have been identified. The presence of SNP rs4244285 and rs 12768009 both reduces nevirapine clearance by approximately $30 \%$ more in African and Asian populations than in Caucasians and Hispanics. ${ }^{12}$ Few clinical pharmacogenetic outcome studies are available, nonetheless evidence shows that the presence of SNP rs2032582 is associated with an improvement in CD4+ count when taking nevirapine. ${ }^{14}$

\section{Protease inhibitors}

Lopinavir and ritonavir are metabolised by the CYP3A4 and CYP3A5 iso enzymes. Genetic polymorphisms in the genes coding for these enzymes (rs4149056) have been attributed to differences in lopinavir/ritonavir plasma concentrations in European and Asian populations, thereby influencing a patient's response to treatment. These include the development of adverse effects such as hepatotoxicity, dyslipidaemia and gastrointestinal symptoms. ${ }^{33}$ A recent South African study evaluating the same reported polymorphism was unfortunately unable to demonstrate any significant association between lopinavir plasma levels and the presence of this variation in African patients. ${ }^{34}$

Atazanavir is mostly indicated as a second line alternative in HIV patients who are unable to tolerate ritonavir. Its metabolism is mainly determined by CYP3A4 activity, which in turn is regulated by the pregnane $X$ receptor (PXR). ${ }^{35}$ The presence of a SNP ( $r$ 2472677) associated with the expression of PXR results in a $17 \%$ increase in atazanavir clearance, therefore resulting in potentially sub-optimal plasma concentrations. Unlike the majority of studies where significant differences in metabolism are observed between populations harbouring the same polymorphism, demographic factors do not seem to have an effect on the clearance of atazanavir, and could therefore be more accurately predicted in individuals carrying the variant allele. $^{36}$

\section{The way forward}

A few commercial South African genetic laboratories have seized the opportunity to offer custom genetic screening tests (or panels) with regard to associated single nucleotide variations which may be encountered in specific disease states. Self-funded customers now have the convenience of having their DNA screened according to a selected panel to determine their genetic susceptibility to some limited (but common) diseases. Some of these include cardiovascular disease, cancer and obesity risk, psychiatric conditions, nutritional requirements and response to certain medication. The benefits of pharmacogenomic testing are well documented in preventing the incidence of adverse drug reactions, improving the efficacy of treatment, decreasing the treatment duration and cost saving on ineffective medications. ${ }^{37}$ However, there undoubtedly exists a need to include African groups in genomics research to identify local variants of pharmacogenomic significance, since it is evident from comparative studies that not all identified polymorphisms are applicable to the majority of the South African population.

Similarly, genomic screening of HIV patients prior to initiating ARV treatment may determine their risk for developing serious adverse effects, therefore individualising treatment may provide a useful cost-saving benefit to the overburdened public health care system. More local pharmacogenetic studies are required to determine the frequency of those variants currently reported in the international literature for its applicability to the South African population. Likewise, additional pharmacogenetic parameters, such as clinical response to treatment or the ability to predict treatment failure, should be investigated. Further robust clinical evidence and cost-effectiveness studies specific to our diverse rainbow population are necessary to consider the possible routine adoption of pharmacogenetic tests in order to make recommendations regarding certain aspects of HIV treatment in South Africa.

\section{References}

1. DeLisi $C$. The Human Genome Project: the ambitious proposal to map and decipher the complete sequence of human DNA. Am Sci. 1988;76(5):488-93.

2. Hood L, Galas D. The digital code of DNA. Nature. 2003;421(6921):444-8. DOI:10.1038/nature01410.

3. Di Sanzo M, Cipolloni L, Borro M, La Russa R, Santurro A, Scopetti $M$, et al. Clinical Applications of Personalized Medicine: A New Paradigm and Challenge. Curr Pharm Biotechnol. 2017;18(3):194-203. doi: $10.2174 / 1389201018666170224105600$

4. Reardon S. Precision-medicine plan raises hopes: US initiative highlights growing focus on targeted therapies. Nature. 2015;517(7536):540-1.

5. Dickmann LJ, Ware JA. Pharmacogenomics in the age of personalized medicine. Drug Discov Today Technol. 2016;21-22:11-6. PMCID:27978982. doi: 10.1016/j. ddtec.2016.11.003

6. Zanger UM, Schwab M. Cytochrome P450 enzymes in drug metabolism: regulation of gene expression, enzyme activities, and impact of genetic variation. Pharmacol Ther. 2013;138(1):103-41.

7. Cotterman CW. Regular two-allele and three-allele phenotype systems. Am J Hum Genet. 1953;5(3):193-235. PMCID:PMC1716480.

8. Gray KA, Yates B, Seal RL, Wright MW, Bruford EA. Genenames.org: the HGNC resources in 2015. Nucleic Acids Res. 2015;43(Database issue):D1079-85. PMCID:PMC4383909. doi: 10.1093/nar/gku1071.

9. Wilkinson GR. Drug metabolism and variability among patients in drug response. N Engl J Med. 2005;352(21):2211-21. PMCID:15917386. doi: 10.1056/ NEJMra032424

10. Phillips KA, Veenstra DL, Oren E, Lee JK, Sadee W. Potential role of pharmacogenomics in reducing adverse drug reactions: a systematic review. JAMA. 2001;286(18):2270-9. PMCID:11710893. doi: 10.1001/jama.286.18.2270

11. Kwara A, Lartey M, Sagoe KW, Rzek NL, Court MH. CYP2B6 (c.516G-->T) and CYP2A6 ( ${ }^{*} 9 B$ and/or $\left.{ }^{*} 17\right)$ polymorphisms are independent predictors of efavirenz plasma concentrations in HIV-infected patients. Br J Clin Pharmacol. 2009;67(4):427-36. PMCID:PMC2679106. doi: 10.1111/j.1365-2125.2009.03368.x

12. Lehr T, Yuan J, Hall D, Zimdahl-Gelling $H$, Schaefer $H G$, Staab $A$, et al. Integration of absorption, distribution, metabolism, and elimination genotyping data into a population pharmacokinetic analysis of nevirapine. Pharmacogenet Genomics. 2011;21(11):721-30. PMCID:21860339. doi: 10.1097/FPC.0b013e32834a522e

13. Fellay J, Shianna KV, Ge D, Colombo S, Ledergerber B, Weale M, et al. A whole-genome association study of major determinants for host control of HIV-1. Science. 2007;317(5840):944-7. PMCID:PMC1991296. doi: 10.1126/ science. 1143767 
14. Zhu P, Zhu Q, Zhang Y, Ma X, Li Z, Li J, et al. ABCB1 variation and treatment response in AIDS patients: initial results of the Henan cohort. PLoS One. 2013;8(1):e55197. PMCID:PMC3555879. doi: 10.1371/journal.pone.0055197

15. Ghodke Y, Anderson PL, Sangkuhl K, Lamba J, Altman RB, Klein TE. PharmGKB summary: zidovudine pathway. Pharmacogenet Genomics. 2012;22(12):891-4. PMCID:PMC3696524. doi: 10.1097/FPC.0b013e32835879a8

16. Quirk E, McLeod H, Powderly W. The pharmacogenetics of antiretroviral therapy: a review of studies to date. Clin Infect Dis. 2004;39(1):98-106. PMCID:15206060. doi: $10.1086 / 421557$

17. Rajman I, Knapp L, Morgan T, Masimirembwa C. African Genetic Diversity: Implications for Cytochrome P450-mediated Drug Metabolism and Drug Development. EBioMedicine. 2017;17:67-74. PMCID:PMC5360579. doi: 10.1016/j. ebiom.2017.02.017

18. Barco E, Nóvoa S. The pharmacogenetics of HIV treatment: a practical clinical approach. J Pharmacogenomics Pharmacoproteomics. 2013;4(1):21530645.1000116. doi: 10.4172/2153-0645.100016

19. Tozzi V. Pharmacogenetics of antiretrovirals. Antiviral Res. 2010;85(1):190-200. doi: 10.1016/j.antiviral.2009.09.001

20. Meintjes G, Moorhouse MA, Carmona S, Davies N, Dlamini S, Van Vuuren C, et al. Adult antiretroviral therapy guidelines 2017. S Afr J HIV Med. 2017;18(1).

21. South African Government [Internet]. National consolidated guidelines for the prevention of mother-to-child transmission of HIV (PMTCT) and the management of HIV in children, adolescents and adults. National Department of Health; 2015 [updated 2015 April; accessed on 04 Jan 2019]. Available from: https://sahivsoc.org/Files/ART\%20Guidelines\%2015052015.pdf.

22. Boulle A, Van Cutsem G, Hilderbrand K, Cragg C, Abrahams M, Mathee S, et al. Seven-year experience of a primary care antiretroviral treatment programme in Khayelitsha, South Africa. Aids. 2010;24(4):563-72. PMCID:20057311. doi: 10.1097/QAD.0b013e328333bfb7

23. Kearney BP, Flaherty JF, Shah J. Tenofovir disoproxil fumarate: clinical pharmacology and pharmacokinetics. Clin Pharmacokinet. 2004;43(9):595-612. PMCID:15217303. doi: 10.2165/00003088-200443090-00003

24. Abla N, Chinn LW, Nakamura T, Liu L, Huang CC, Johns SJ, et al. The human multidrug resistance protein 4 (MRP4, $A B C C 4)$ : functional analysis of a highly polymorphic gene. J Pharmacol Exp Ther. 2008;325(3):859-68. PMCID:PMC2612728. doi: 10.1124/jpet.108.136523

25. Stevens RC, Blum MR, Rousseau FS, Kearney BP. Intracellular pharmacology of emtricitabine and tenofovir. Clin Infect Dis. 2004;39(6):877-8. doi: $10.1086 / 423810$

26. Kim MY, Ives DH. Human deoxycytidine kinase: kinetic mechanism and end product regulation. Biochemistry. 1989;28(23):9043-7. PMCID:2557916. doi: 10.1021/bi00449a012
27. Lee HW, Lee SH, Lee MG, Ahn SH, Chang HY, Han KH. The clinical implication of single nucleotide polymorphisms in deoxycytidine kinase in chronic hepatitis $B$ patients treated with lamivudine. J Med Virol. 2016;88(5):820-7. PMCID:26400223 doi: 10.1002/jmv.24393

28. Veal GJ, Back DJ. Metabolism of Zidovudine. Gen Pharmacol. 1995;26(7):1469-75. PMCID:8690233. doi: 10.1016/0306-3623(95)00047-X

29. Kwara A, Lartey M, Boamah I, Rezk NL, Oliver-Commey J, Kenu E, et al Interindividual variability in pharmacokinetics of generic nucleoside reverse transcriptase inhibitors in TB/HIV-coinfected Ghanaian patients: UGT2B7* $1 \mathrm{c}$ is associated with faster zidovudine clearance and glucuronidation. J Clin Pharmacol. 2009;49(9):1079-90. doi: 10.1177/0091270009338482

30. de Bethune MP. Non-nucleoside reverse transcriptase inhibitors (NNRTIs), their discovery, development, and use in the treatment of HIV-1 infection: a review of the last 20 years (1989-2009). Antiviral Res. 2010;85(1):75-90. doi: 10.1016/j. antiviral.2009.09.008

31. Ogburn ET, Jones DR, Masters AR, Xu C, Guo Y, Desta Z. Efavirenz primary and secondary metabolism in vitro and in vivo: identification of novel metabolic pathways and cytochrome P450 2A6 as the principal catalyst of efavirenz 7-hydroxylation. Drug Metab Dispos. 2010;38(7):1218-29. PMCID:PMC2908985. doi: $10.1124 /$ dmd. 109.031393

32. Sinxadi PZ, Leger PD, Mcllleron HM, Smith PJ, Dave JA, Levitt NS, et al. Pharmacogenetics of plasma efavirenz exposure in HIV-infected adults and children in South Africa. Br J Clin Pharmacol. 2015;80(1):146-56. PMCID:PMC4500334. doi: 10.1111/bcp.12590

33. Schipani A, Egan D, Dickinson L, Davies G, Boffito M, Youle M, et al. Estimation of the effect of SLCO1B1 polymorphisms on lopinavir plasma concentration in HIV-infected adults. Antivir Ther. 2012;17(5):861-8. PMCID:PMC3443796. doi: 10.3851/imp2095

34. Mpeta B, Kampira E, Castel S, Mpye KL, Soko ND, Wiesner L, et al. Differences in genetic variants in lopinavir disposition among HIV-infected Bantu Africans. Pharmacogenomics. 2016;17(7):679-90. doi: 10.2217/pgs.16.14

35. Mbatchi LC, Brouillet JP, Evrard A. Genetic variations of the xenoreceptors NR112 and NR1/3 and their effect on drug disposition and response variability. Pharmacogenomics. 2018;19(1):61-77. PMCID:29199543. doi: 10.2217/ pgs-2017-0121

36. Schipani A, Siccardi M, D'Avolio A, Baietto L, Simiele M, Bonora S, et al. Population pharmacokinetic modeling of the association between $63396 \mathrm{C}$ $>$ T pregnane $\mathrm{X}$ receptor polymorphism and unboosted atazanavir clearance. Antimicrob Agents Chemother. 2010;54(12):5242-50. PMCID:PMC2981241. doi: 10.1128/aac.00781-10

37. Plumpton CO, Roberts D, Pirmohamed M, Hughes DA. A Systematic review of economic evaluations of pharmacogenetic testing for prevention of adverse drug reactions. Pharmacoeconomics. 2016;34(8):771-93. doi: 10.1007/ s40273-016-0397-9 